UDC 539

\title{
Ion drift in parent gas for cesium, rubidium, and mercury
}

\author{
R.I. Golyatina, S.A. Maiorov* \\ Prokhorov General Physics Institute of the Russian Academy of Sciences, \\ 38 Vavilov Str., 119991 Moscow, Russia \\ *e-mail:mayorov_sa@mail.ru
}

\begin{abstract}
The results of Monte Carlo calculations of the ion drift characteristics in a constant and homogeneous electric field are presented for the values of the applied intensity in the range $1<\mathrm{E} / \mathrm{N}<1000 \mathrm{Td}$ for cesium, rubidium and mercury. The results of molecular dynamics simulation are used together with the Monte Carlo method for calculation of collisional characteristics. The drift velocity, mean kinetic energy, longitudinal and transverse diffusion coefficients, mean free path, and the fraction of collisions with backward scattering are calculated. It is shown that the introduction of dimensionless units makes it possible to reduce the characteristics for different gases to universal curves. And the Bhatnagar, Gross, and Krook collision integral for the problem of ion drift in an own gas leads to significant errors. Also, an unexpected and nontrivial fact about the collisions with backward scattering is obtained and conclusions provided. It is found that using the BGK collision integral foe deseviling of ion drift an own gas leads to significant errors. There are some discussions regarding the large difference between present calculations and data of the BGK theory.
\end{abstract}

PACS number(s): 51.50.+v,51.10.+y,52.80.Dy,52.25.Fi

Keywords: ion, parent gas, vapor, drift, collision, hard-sphere model, cross-section, BGK model.

\section{Introduction}

The flow of ions in a constant homogeneous electric field is usually characterized by the drift velocity, the values of the average energies of ions, with coefficients of mobility and diffusion in the longitudinal and transverse directions. The velocity of the ion drift in a gas under the action of a constant homogeneous electric field is given by the relation

$$
W=\mu E,
$$

where the coefficient of ion mobility $\mu(E, N, T)$ generally depends on both the field strength and gas parameters (temperature $T$, pressure $p=N T$, composition). A large number of experimental studies have been devoted to the determination of the diffusion coefficients and the mobility of ions in gases, and it can be assumed that in weak and moderately strong fields $(E / N<1000 T d)$ the ion mobility is known with a very high accuracy $<1 \%[1-4]$.

In the handbooks, experimental and calculated data are usually presented only the drift velocity, but other kinetic characteristics of the ion drift are necessary for analyzing the properties of the gas discharge. For example, to determine the ion Debye radius, it is necessary to know the average ion energy, taking into account their heating in an electric field. Many characteristics of a gas discharge are determined from the ionic distribution of velocities, and knowledge of the ion velocity distribution function is often sufficient.

In [5], a modeling technique used to calculate the ion drift characteristics in a gas is described. The results of calculations of the characteristics of ion drift in a constant and homogeneous electric field at $1<\mathrm{E} / \mathrm{N}<1000$ for all noble gases. In this paper, which is a continuation of $[6,7]$, the results of molecular dynamics calculations are presented together with the Monte Carlo procedure for collision simulation. The characteristics of ion drift in a constant and homogeneous electric field at $1<\mathrm{E} / \mathrm{N}<1000 \mathrm{Td}$ for cesium, rubidium and mercury are calculated.

\section{Theory}

In Table. 1 - 3 shows the flow characteristics of singly charged rubidium, cesium and mercury ions during their drift in a constant and homogeneous electric field in their own gas at atom temperature $300 \mathrm{~K}$ and atomic density $2.69 * 10^{19} \mathrm{~cm}^{-3}$. In addition to the drift velocity, the tables show: the effective temperature of the ions, which is related to the 
average kinetic energy of the ions by the ratio $\langle\varepsilon\rangle=\frac{1}{2} m\left\langle\mathrm{v}^{2}\right\rangle=\frac{3}{2} T_{\text {eff }}$; temperature of ions along the field $T_{\|}$and across the field $T_{\perp}$, so the average energy of the ion is equal to $\langle\varepsilon\rangle=\frac{1}{2} m W^{2}+\frac{1}{2} T_{\|}+T_{\perp}$; the diffusion coefficients in the direction along and across the field, the mean free path, and the fraction of collisions with backward scattering with respect to the total number of collisions. The exact values of the various characteristics at intermediate points can be obtained by interpolation.

Table 1 - Characteristics of the cesium ion flow during their drift in a constant and homogeneous electric field in their own gas at $300 \mathrm{~K}$ and atomic density $2.6910^{19} / \mathrm{cm}^{3}$.

\begin{tabular}{|c|c|c|c|c|c|c|c|c|}
\hline $\begin{array}{c}\mathrm{E} / \mathrm{N}, \\
\mathrm{Td}\end{array}$ & $\begin{array}{c}\mathrm{W}, \\
\mathrm{km} / \mathrm{s}\end{array}$ & $\begin{array}{c}T_{\text {eff }}, \\
\mathrm{K}\end{array}$ & $\begin{array}{c}T_{\square}, \\
\mathrm{K}\end{array}$ & $\begin{array}{c}T_{\perp}, \\
\mathrm{K}\end{array}$ & $\begin{array}{c}D_{l} \\
\mathrm{~cm}^{2} / \mathrm{s}\end{array}$ & $\begin{array}{c}D_{t} \\
\mathrm{~cm}^{2} / \mathrm{s}\end{array}$ & $\begin{array}{c}\text { m.f.p. } \\
\text { HM }\end{array}$ & $\begin{array}{c}\text { BS } \\
(\mathrm{BS}+\text { Iso })\end{array}$ \\
\hline 1 & 0.0006 & 300.2 & 300.3 & 300.2 & 0.0056 & 0.0056 & 3.8 & 0.093 \\
\hline 2 & 0.0012 & 300.2 & 300.3 & 300.2 & 0.0056 & 0.0056 & 3.8 & 0.093 \\
\hline 5 & 0.0031 & 300.3 & 300.4 & 300.2 & 0.0056 & 0.0056 & 3.8 & 0.093 \\
\hline 10 & 0.0062 & 300.4 & 300.7 & 300.3 & 0.0056 & 0.0056 & 3.8 & 0.093 \\
\hline 20 & 0.0124 & 301.1 & 302.3 & 300.5 & 0.0056 & 0.0056 & 3.8 & 0.094 \\
\hline 50 & 0.0310 & 306.1 & 313.7 & 302.2 & 0.0056 & 0.0056 & 3.8 & 0.095 \\
\hline 100 & 0.0613 & 323.1 & 353.4 & 308.0 & 0.0057 & 0.0056 & 3.9 & 0.098 \\
\hline 200 & 0.119 & 384.3 & 496.7 & 328.0 & 0.0060 & 0.0058 & 4.2 & 0.11 \\
\hline 500 & 0.259 & 676.7 & 1199 & 415.3 & 0.0068 & 0.0063 & 5.4 & 0.16 \\
\hline 1000 & 0.431 & 1259 & 2649 & 563.8 & 0.0078 & 0.0068 & 6.9 & 0.24 \\
\hline
\end{tabular}

Table 2 - Characteristics of the rubidium ion flow during their drift in a constant and homogeneous electric field in their own gas at $300 \mathrm{~K}$ and the density of atoms $2.6910^{19} / \mathrm{cm}^{3}$.

\begin{tabular}{|c|c|c|c|c|c|c|c|c|}
\hline $\begin{array}{c}\mathrm{E} / \mathrm{N}, \\
\mathrm{Td}\end{array}$ & $\begin{array}{c}\mathrm{W}, \\
\mathrm{km} / \mathrm{s}\end{array}$ & $\begin{array}{c}T_{\text {eff }}, \\
\mathrm{K}\end{array}$ & $\begin{array}{c}T_{\square}, \\
\mathrm{K}\end{array}$ & $\begin{array}{c}T_{\perp}, \\
\mathrm{K}\end{array}$ & $\begin{array}{c}D_{l} \\
\mathrm{~cm}^{2} / \mathrm{s}\end{array}$ & $\begin{array}{c}D_{t} \\
\mathrm{~cm}^{2} / \mathrm{s}\end{array}$ & $\begin{array}{c}\text { m.f.p. } \\
\mathrm{nm}\end{array}$ & $\begin{array}{c}\text { BS } \\
(\mathrm{BS}+\text { Iso) }\end{array}$ \\
\hline 1 & 0.00041 & 300.2 & 300.4 & 300.2 & 0.0038 & 0.0038 & 3.3 & 0.11 \\
\hline 2 & 0.00084 & 300.2 & 300.4 & 300.2 & 0.0038 & 0.0038 & 3.3 & 0.11 \\
\hline 5 & 0.00212 & 300.2 & 300.4 & 300.2 & 0.0038 & 0.0038 & 3.3 & 0.11 \\
\hline 10 & 0.0043 & 300.4 & 300.5 & 300.3 & 0.0038 & 0.0038 & 3.3 & 0.11 \\
\hline 20 & 0.0085 & 300.9 & 301.7 & 300.5 & 0.0038 & 0.0038 & 3.3 & 0.11 \\
\hline 50 & 0.0213 & 304.5 & 310.3 & 301.7 & 0.0039 & 0.0038 & 3.3 & 0.11 \\
\hline 100 & 0.0423 & 317.2 & 340.0 & 305.8 & 0.0039 & 0.0038 & 3.4 & 0.12 \\
\hline 200 & 0.0822 & 363.5 & 449.2 & 320.6 & 0.0040 & 0.0039 & 3.6 & 0.13 \\
\hline 500 & 0.183 & 594.3 & 1006 & 388.5 & 0.0045 & 0.0042 & 4.5 & 0.17 \\
\hline 1000 & 0.307 & 1070 & 2192 & 508.6 & 0.0051 & 0.0044 & 5.6 & 0.24 \\
\hline
\end{tabular}


Table 3 - Characteristics of the mercury ion flow during their drift in a constant and homogeneous electric field in their own gas at $300 \mathrm{~K}$ and atomic density $2.6910^{19} / \mathrm{cm}^{3}$.

\begin{tabular}{|c|c|c|c|c|c|c|c|c|}
\hline $\begin{array}{c}\mathrm{E} / \mathrm{N}, \\
\mathrm{Td}\end{array}$ & $\begin{array}{c}\mathrm{W}, \\
\mathrm{km} / \mathrm{s}\end{array}$ & $\begin{array}{c}T_{\text {eff }}, \\
\mathrm{K}\end{array}$ & $\begin{array}{c}T_{\square}, \\
\mathrm{K}\end{array}$ & $\begin{array}{c}T_{\perp}, \\
\mathrm{K}\end{array}$ & $\begin{array}{c}D_{l} \\
\mathrm{~cm}^{2} / \mathrm{s}\end{array}$ & $\begin{array}{c}D_{t} \\
\mathrm{~cm}^{2} / \mathrm{s}\end{array}$ & $\begin{array}{c}\mathrm{m} . \text { f.p. } \\
\mathrm{nm}\end{array}$ & $\begin{array}{c}\text { BS / } \\
\text { (BS+Iso) }\end{array}$ \\
\hline 1 & 0.0006 & 300.2 & 300.3 & 300.1 & 0.0055 & 0.0055 & 8.0 & 0.36 \\
\hline 2 & 0.0012 & 300.2 & 300.3 & 300.1 & 0.0055 & 0.0055 & 8.0 & 0.36 \\
\hline 5 & 0.0032 & 300.3 & 300.5 & 300.2 & 0.0055 & 0.0055 & 8.0 & 0.36 \\
\hline 10 & 0.0063 & 300.7 & 301.5 & 300.3 & 0.0055 & 0.0055 & 8.0 & 0.36 \\
\hline 20 & 0.0127 & 302.4 & 305.6 & 300.7 & 0.0055 & 0.0055 & 8.0 & 0.36 \\
\hline 50 & 0.0313 & 313.4 & 333.5 & 303.3 & 0.0056 & 0.0055 & 8.2 & 0.37 \\
\hline 100 & 0.061 & 348.4 & 422.7 & 311.3 & 0.0057 & 0.0055 & 8.6 & 0.38 \\
\hline 200 & 0.113 & 457.8 & 704.6 & 334.4 & 0.0062 & 0.0054 & 9.6 & 0.41 \\
\hline 500 & 0.243 & 969.3 & 2046 & 430.8 & 0.0099 & 0.0058 & 12.7 & 0.48 \\
\hline 1000 & 0.441 & 2457 & 5937 & 716.4 & 0.0257 & 0.0085 & 18.3 & 0.53 \\
\hline
\end{tabular}

The numerical data given above make it possible to obtain a fairly complete picture of the character of ion drift. We note some features of ion velocity distributions:

1) Analysis of the distribution functions shows that when ion drift, in conditions typical for gasdischarge plasma conditions, there is an appreciable heating of the ions not only in the longitudinal direction, but also in the transverse direction. This is due to close collisions, when besides recharging, scattering also occurs at an arbitrary direction in the center-of-mass system;

2) Even at a sufficiently high reduced electric field strength an ion flow does not form supersonic flux, in which the velocity of thermal, chaotic motion would be less than the velocity of the directed motion - the drift velocity. As already noted, due to the fact that with increasing field strength simultaneously with the increase of drift velocity there is an increase in the dispersion of the distribution function of the ion velocity (i.e., the longitudinal and transverse temperatures);

3 ) In the velocity distribution of ions there is a large anisotropy (and a large difference between the longitudinal and transverse temperatures $\mathrm{T}_{\|}$and $T_{\perp}$ ). Therefore, the diffusion fluxes along and across the field can vary greatly in magnitude;

4) From the analysis of ion velocity distribution functions, from the analysis of other characteristics of the ion flux it follows that velocity module distribution, as well as the velocity projections, has significant differences from the corresponding Maxwellian distributions. Moreover, there is a difference not only in the tails of the distribution functions, but also in the central part. Accordingly, the distribution function of ion velocity during their drift in their own gas cannot be described by a Gaussian curve (shifted Maxwell distribution function).

In this connection, calculations were made with the determination of the fraction of collisions with backward scattering with respect to the total number of collisions, depending on the reduced field strength (naturally, the number of collisions does not include collisions with scattering at small angles due to divergence of the corresponding collision integral). The results of the calculations showed that there is an unexpected fact - although the cross sections with charge exchange are the largest, collisions with scattering back are only 15 - 45 percent. This takes place not only for the ions considered here for cesium, rubidium and mercury, but also for noble gases [7].

An analysis of the results of calculations shows that even in a strong field collisions with backward scattering are not dominant. But it is generally accepted that there is a decisive influence of collisions with the transfer of a charge, and often, when considering ion drift, only collisions with charge exchange are taken into account.

To take into account the effect of collisions on the velocity distribution of ions, the model collision integral of Bhatnagar, Gross, and Krook (integral BGK) is often used [6, 8-11]. However, the ion flow characteristics given in the calculations show a large deviation from the equilibrium distribution and a significant effect of collisions with isotropic scattering. In this connection, let us consider, as an example, the result of using BGK of the collision integral 
Table 3 - Characteristics of the mercury ion flow during their drift in a constant and homogeneous electric field in their own gas at $300 \mathrm{~K}$ and atomic density $2.6910^{19} / \mathrm{cm}^{3}$.

\begin{tabular}{|c|c|c|c|c|c|c|c|c|}
\hline $\begin{array}{c}\mathrm{E} / \mathrm{N}, \\
\mathrm{Td}\end{array}$ & $\begin{array}{c}\mathrm{W}, \\
\mathrm{km} / \mathrm{s}\end{array}$ & $\begin{array}{c}T_{\text {eff }}, \\
\mathrm{K}\end{array}$ & $\begin{array}{c}T_{\square}, \\
\mathrm{K}\end{array}$ & $\begin{array}{c}T_{\perp}, \\
\mathrm{K}\end{array}$ & $\begin{array}{c}D_{l} \\
\mathrm{~cm}^{2} / \mathrm{s}\end{array}$ & $\begin{array}{c}D_{t} \\
\mathrm{~cm}^{2} / \mathrm{s}\end{array}$ & $\begin{array}{c}\mathrm{m} . \text { f.p. } \\
\mathrm{nm}\end{array}$ & $\begin{array}{c}\text { BS / } \\
\text { (BS+Iso) }\end{array}$ \\
\hline 1 & 0.0006 & 300.2 & 300.3 & 300.1 & 0.0055 & 0.0055 & 8.0 & 0.36 \\
\hline 2 & 0.0012 & 300.2 & 300.3 & 300.1 & 0.0055 & 0.0055 & 8.0 & 0.36 \\
\hline 5 & 0.0032 & 300.3 & 300.5 & 300.2 & 0.0055 & 0.0055 & 8.0 & 0.36 \\
\hline 10 & 0.0063 & 300.7 & 301.5 & 300.3 & 0.0055 & 0.0055 & 8.0 & 0.36 \\
\hline 20 & 0.0127 & 302.4 & 305.6 & 300.7 & 0.0055 & 0.0055 & 8.0 & 0.36 \\
\hline 50 & 0.0313 & 313.4 & 333.5 & 303.3 & 0.0056 & 0.0055 & 8.2 & 0.37 \\
\hline 100 & 0.061 & 348.4 & 422.7 & 311.3 & 0.0057 & 0.0055 & 8.6 & 0.38 \\
\hline 200 & 0.113 & 457.8 & 704.6 & 334.4 & 0.0062 & 0.0054 & 9.6 & 0.41 \\
\hline 500 & 0.243 & 969.3 & 2046 & 430.8 & 0.0099 & 0.0058 & 12.7 & 0.48 \\
\hline 1000 & 0.441 & 2457 & 5937 & 716.4 & 0.0257 & 0.0085 & 18.3 & 0.53 \\
\hline
\end{tabular}

The numerical data given above make it possible to obtain a fairly complete picture of the character of ion drift. We note some features of ion velocity distributions:

1) Analysis of the distribution functions shows that when ion drift, in conditions typical for gasdischarge plasma conditions, there is an appreciable heating of the ions not only in the longitudinal direction, but also in the transverse direction. This is due to close collisions, when besides recharging, scattering also occurs at an arbitrary direction in the center-of-mass system;

2) Even at a sufficiently high reduced electric field strength an ion flow does not form supersonic flux, in which the velocity of thermal, chaotic motion would be less than the velocity of the directed motion - the drift velocity. As already noted, due to the fact that with increasing field strength simultaneously with the increase of drift velocity there is an increase in the dispersion of the distribution function of the ion velocity (i.e., the longitudinal and transverse temperatures);

3 ) In the velocity distribution of ions there is a large anisotropy (and a large difference between the longitudinal and transverse temperatures $\mathrm{T}_{\|}$and $T_{\perp}$ ). Therefore, the diffusion fluxes along and across the field can vary greatly in magnitude;

4) From the analysis of ion velocity distribution functions, from the analysis of other characteristics of the ion flux it follows that velocity module distribution, as well as the velocity projections, has significant differences from the corresponding Maxwellian distributions. Moreover, there is a difference not only in the tails of the distribution functions, but also in the central part. Accordingly, the distribution function of ion velocity during their drift in their own gas cannot be described by a Gaussian curve (shifted Maxwell distribution function).

In this connection, calculations were made with the determination of the fraction of collisions with backward scattering with respect to the total number of collisions, depending on the reduced field strength (naturally, the number of collisions does not include collisions with scattering at small angles due to divergence of the corresponding collision integral). The results of the calculations showed that there is an unexpected fact - although the cross sections with charge exchange are the largest, collisions with scattering back are only 15 - 45 percent. This takes place not only for the ions considered here for cesium, rubidium and mercury, but also for noble gases [7].

An analysis of the results of calculations shows that even in a strong field collisions with backward scattering are not dominant. But it is generally accepted that there is a decisive influence of collisions with the transfer of a charge, and often, when considering ion drift, only collisions with charge exchange are taken into account.

To take into account the effect of collisions on the velocity distribution of ions, the model collision integral of Bhatnagar, Gross, and Krook (integral BGK) is often used [6, 8-11]. However, the ion flow characteristics given in the calculations show a large deviation from the equilibrium distribution and a significant effect of collisions with isotropic scattering. In this connection, let us consider, as an example, the result of using BGK of the collision integral 
for analyzing the characteristics of the ion flux, taking into account only collisions with resonant charge exchange $[7,8]$.

The characteristics of the ion flux can be determined by solving the Boltzmann kinetic equation for the ion distribution function $f(\mathrm{v})$ :

$$
\frac{\partial f}{\partial t}+\mathrm{v} \nabla f+\frac{e E}{m} \frac{\partial f}{\partial \mathrm{v}}=I_{s t}(f)
$$

where $\mathrm{e}$ - charge, $\mathrm{m}$ - ion mass, $I_{s t}(f)-$ collision integral.

In a weakly ionized plasma, it is often possible to neglect elastic collisions of ions with atoms, electrons, and ions. Since in the case of collisions of ions with the atoms of the proper gas, the cross section for the resonant charge exchange of an ion is usually greatest, then we consider the kinetic equation (2) in the spatially homogeneous case with a constant electric field, taking into account only the resonant charge exchange of ions:

$$
\frac{e E}{m} \frac{\partial f}{\partial u}=\int\left[f\left(\mathrm{v}^{\prime}\right) \varphi(\mathrm{v})-f(\mathrm{v}) \varphi\left(\mathrm{v}^{\prime}\right)\right]\left|\mathrm{v}-\mathrm{v}^{\prime}\right| \sigma_{r e s} n_{a} d \mathrm{v}^{\prime},
$$

where $\mathrm{u}$ - component of velocity along the direction of the electric field, $\sigma_{\text {res }}$ - the cross section of resonant charge exchange, $n_{a}$ - atomic density, The distribution functions of ions and atoms are normalized to unity: $\int f(\mathrm{v}) d \mathrm{v}=\int \varphi(\mathrm{v}) d \mathrm{v}=1$. Equation (3) describes the process of ion transport, which is a relay character - this model was suggested by L. A. Sena $[8,12]$. According to this model, the ion velocity after the collision is equal to the velocity of the atom with which it collided. This model does not take into account the change in the velocity of the atom during the collision.

Let us consider the model of ion motion in the case of the fulfillment of two conditions:

1) The drift velocity $W$ considerably exceeds the thermal velocity of the atoms $W>V_{T}=\left(T_{i} / \mathrm{m}\right)^{1 / 2}$;

2) There are only collisions of one type-with a resonant charge exchange of ions on the atoms of the own gas.

When these conditions are met, we can neglect the thermal motion of the atoms and assume that the ions move uniformly accelerated in a constant electric field $E>0$, stopping after each collision event. The condition $W>V_{T}$ can be satisfied with good accuracy either in the case of a high electric field strength or at a low gas temperature.

In neglecting the thermal energy of the atoms and taking into account only collisions with charge exchange, the Boltzmann kinetic equation has the form $[7,8,12]$ :

$$
\begin{gathered}
\frac{e E}{m} \frac{\partial f}{\partial u}=-f \frac{u}{\lambda_{s t}}, f(u<0)=0 \\
f(0)=c_{1} .
\end{gathered}
$$

If the cross section of resonant charge exchange depends on the velocity, then the ion distribution function has the form:

$$
f(u)=c_{1} \Theta(u) \exp \left(-\frac{m}{e E} \int_{0}^{u} n_{a} \sigma_{r e s}\left(u^{\prime}\right) u^{\prime} d u^{\prime}\right),
$$

where $\Theta(u)$ - Heaviside function, $\mathrm{c}_{1}$ - the constant, determined from the normalization condition.

If the cross section of resonant charge exchange and the mean free path of the ion $\lambda_{s t}=1 / \sigma_{0} n_{a}$ do not depend on the velocity, then the solution of (4) has the form

$$
f(u)=\Theta(u)\left(\frac{2 m}{\pi T_{E}}\right)^{1 / 2} \exp \left(-\frac{m u^{2}}{2 T_{E}}\right),
$$

where $T_{E} \equiv e E \lambda_{s t}$. The distribution (6) is half the Maxwell distribution with a temperature equal to the energy recruited by the ion at the mean free path. Consequently, the average kinetic energy of the ions, due to the motion in the direction of the field, is $\frac{1}{2} m\left\langle u^{2}\right\rangle=\frac{1}{2} T_{E}=\frac{1}{2} e E \lambda_{s t}$. The ion flux density for this distribution is $J_{i}=n_{i}\left(2 e E \lambda_{s t} / \pi m\right)^{1 / 2}$, the average ion velocity (drift velocity) is $W=\left(2 e E \lambda_{s t} / \pi m\right)^{1 / 2}=\left(2 T_{E} / \pi m\right)^{1 / 2}$.

Collisions with charge exchange play the most important role, but collisions of other types have a significant effect on the characteristics of ion velocity distribution. In addition to collisions with recharging, an important role can be played by polarization and gas-kinetic collisions. In the case of such collisions, the ion does not stop, but dissipates at the fixed center in the center-of-mass system of the atom-ion. A good approximation of this type of 
collision is the model of hard spheres, i.e. isotropic scattering. Without consideration of these collisions, heating of the ions in the transverse direction can not be taken into account. By analogy with the hydrodynamic approximation, it is often assumed that the drift of ions in a strong field is described by the shifted distribution function of Maxwell:

$$
f_{0}(\overline{\mathrm{v}})=\left(\frac{m}{2 \pi T_{i}}\right)^{3 / 2} \exp \left(-\frac{m\left[(u-W)^{2}+\mathrm{v}^{2}+w^{2}\right]}{2 T_{i}}\right)
$$

This distribution has two parameters - the drift velocity $\mathrm{W}$ and the ion temperature $T_{i}$, which determines the thermal dispersion of the ion velocities $V_{i}=\left(T_{i} / m\right)^{1 / 2}$, here the direction of the field and drift coincides with the $\mathrm{x}$ axis.

To take into account the impact of collisions, the model collision integral of Bhatnagar, Gross, and Kruk (BGK integral) is often used [7-9]:

$$
I_{s t}=\frac{\varphi-f}{\tau_{0}},
$$

which describes the relaxation of the ion distribution function $f$ to the equilibrium distribution function of atoms $\varphi$ with a characteristic relaxation time $\tau_{0}=$ const. The BGK integral qualitatively correctly describes the process of plasma relaxation to equilibrium in the case of a slight deviation from it. But it is inapplicable if the collision frequency of ions with atoms depends on their relative velocity, or the deviation from equilibrium is large. The ion transport equation in the spatially homogeneous case has the form: $V_{E} \partial f / \partial u=\varphi(u)-f(u)$, where $V_{E}=e E \tau_{0} / m$. Its solution has the form:

$$
f_{E}(u)=\frac{1}{V_{E}} \int_{-\infty}^{u} \varphi\left(u^{\prime}\right) \exp \left(-\frac{u-u^{\prime}}{V_{E}}\right) d u^{\prime} .
$$

In the case of a subthermal flow velocity, when the drift velocity $W \leq(T / m)^{1 / 2}$, and the Maxwellian distribution of atoms $\varphi(u)=\left(m / 2 \pi T_{0}\right)^{1 / 2} \exp \left(-m u^{2} / 2 T_{0}\right)$, the solution (2) with the collision integral (8) has the form

$$
f(u)=\varphi(u)\left(1+u V_{E} / V_{T}^{2}\right) .
$$

This distribution coincides with the expansion of the shifted Maxwellian distribution (7) at $V_{E}=W<<V_{T}=(T / m)^{1 / 2}$. As expected, in the case of a small deviation from equilibrium, the use of the model integral BGK gives a reasonable result. In the case of a high ion flow rate $W>V_{T}=\sqrt{T_{0} / m}$ and a Maxwellian distribution of atoms, the distribution (9) has the asymptotics:

$$
f(u)=\frac{\Theta(u)}{V_{E}} \exp \left(-\frac{u}{V_{E}}\right) .
$$

This distribution describes the uniformly accelerated motion of ions in a constant electric field $E>0$, which stop after each collision event, the probability of which does not depend on the velocities of the ion and the atom. This unnatural hybrid model (taking the properties of polarization and resonant collisions) is a consequence of the structure of the integral BGK for large fields, when $W>(T / m)^{1 / 2}$. It does not take into account the difference of the ion velocity from zero after the collision.

If we neglect the thermal motion of the atoms in comparison with the flow velocity and represent the distribution function of atoms in the form $\varphi(u)=\delta(u)$, then the particle profit in the BGK integral has the form $I_{B G K}^{+}=\varphi / \tau_{0}$, the decrease is $I_{B G K}^{-}=f / \tau_{0}$. For collisions with a resonant predissection at a constant cross section, the profit and loss of the particles has, respectively, a form $I_{\text {res }}^{+}=\varphi / \tau_{0}$, a decrease $-I_{\text {res }}^{-}=\sigma_{0} n_{a} u f$. Consequently, the BGK integral even incorrectly conveys the nature of the particle loss at a qualitative level, this explains the radical difference between the distribution (11) and the physically reasonable distributions (6) and (7).

For polarization collisions characterized by a constant mean free path, the particle loss in the BGK integral can be reduced to the form $I_{p o l}^{-}=f / \tau_{0}$. But the profit of particles in the collision integral depends on the entire distribution

function and can in no way be approximated by the quantity $I_{B G K}^{+}=\varphi / \tau_{0}$. This means that, regardless of the form of the distribution function $f(\mathrm{v})$, the 
The BGK integral does not allow to take into account the following factors:

1) for collisions characterized by a constant cross section (charge exchange, gas-kinetic), it does not take into account the dependence of the collision probability on the velocity;

2) for collisions characterized by a constant mean free path (polarization collisions), he does not take into account the difference between the ion velocity after the scattering event from zero.

These factors are decisive at a drift velocity comparable to the thermal velocity of the atoms. Consequently, the BGK integral is inapplicable for the problem of determining the characteristics of the ion drift in its own gas.

Let us compare the results of calculating the drift velocity of cesium, rubidium and mercury ions

$$
\overline{V_{T}}=\overline{\pi^{1 / 2}\left(1+F^{1 / 2}+F\right)} \cdot
$$

\section{Results and discussions}

In Figure 1 shows the results of calculating the drift velocity of an ion in its own gas, depending on the electric field strength for cesium, rubidium and mercury. In Figure 2 shows the same results, but the drift velocity is normalized to the value of the thermal velocity (the velocity of an ion with an energy equal to the temperature of the atoms), and the field is normalized by the value of the characteristic "heating field": $F=E / E_{T}$. The magnitude of the heating field is determined in such a way that an energy equal to the temperature of the atoms is accumulated on the mean free path.

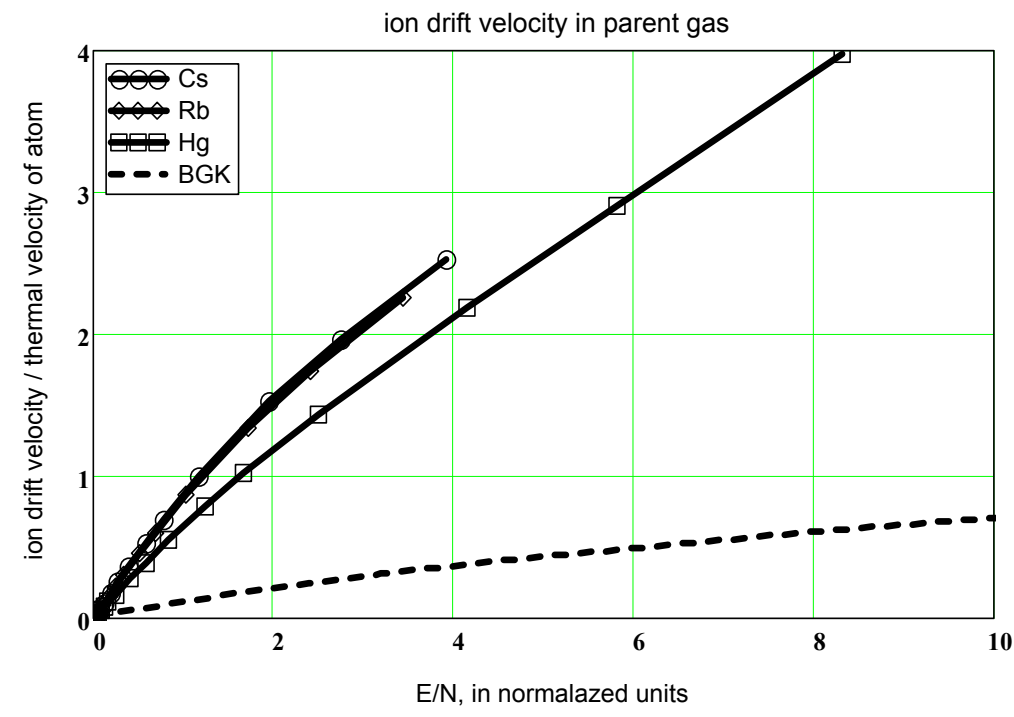

Figure 1 - Results of calculating the drift velocity of an ion in its own gas as a function of the electric field strength in dimensionless units. The drift velocity is normalized to the value of the thermal velocity (the velocity of an ion with an energy equal to the temperature of the atoms), the field is normalized to the value of the characteristic "heating field", in which an energy equal to the temperature of the atoms is accumulated on the mean free path. The shaded curve is the solution of the Boltzmann equation with the BGK collision integral (11). 


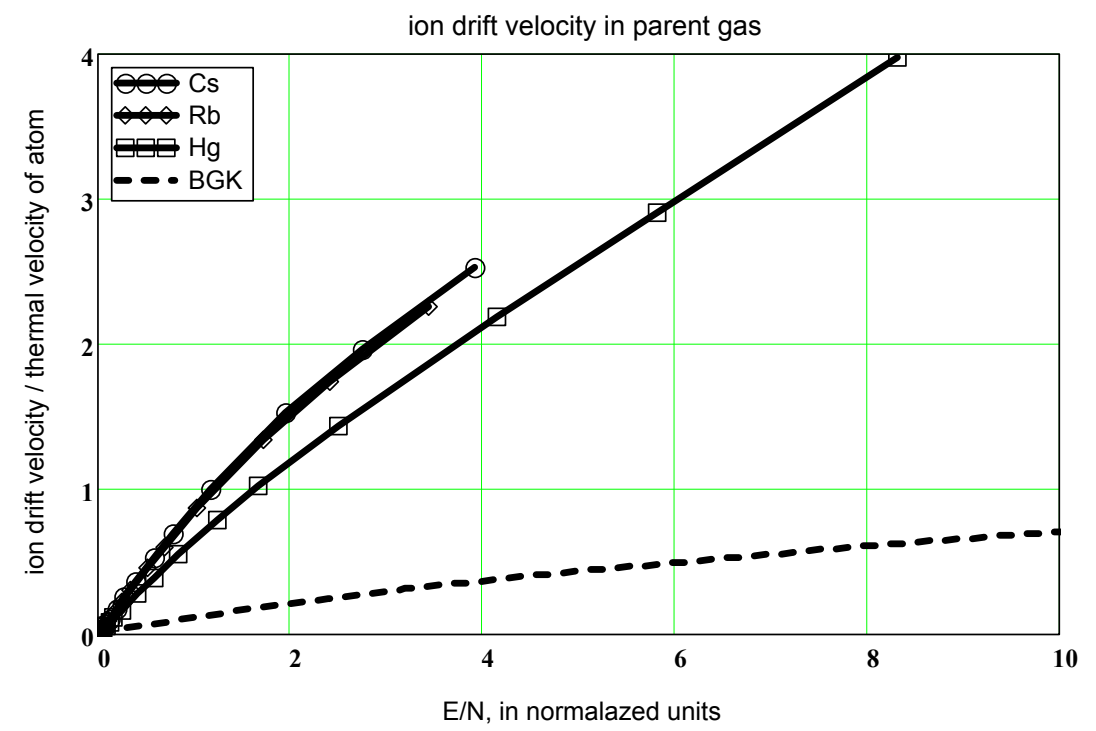

Figure 2 - Results of calculating the drift velocity of an ion in its own gas as a function of the electric field strength in dimensionless units. The drift velocity is normalized to the value of the thermal velocity (the velocity of an ion with an energy equal to the temperature of the atoms), the field is normalized to the value of the characteristic "heating field", in which an energy equal to the temperature of the atoms is accumulated on the mean free path. The shaded curve is the solution of the Boltzmann equation with the BGK collision integral (11)

\section{Conslusions} sions:

The above graphs make the following conclu-

1) The introduction of dimensionless units makes it possible to reduce the characteristics for different gases to universal curves;

2) The BGK collision integral for the problem of ion drift in an own gas leads to significant errors, which does not allow even describing real processes at a qualitative level (see, for example [7-9]);

3) There is an unexpected and nontrivial fact: although cross sections with charge exchange are the largest, collisions with backward scattering make up only $15-45$ percent for noble gases at 300 $\mathrm{K}$ (for this reason see [16], where an attempt was made to approximate collisions in the form of a sum of collisions with isotropic scattering and backscattering).

The analysis showed that such a large difference in the calculations and the BGK theory is due to the fact that even in a strong field, collisions with backward scattering are not dominant. The results of the calculations demonstrate that the approach to the analysis of ion drift based on the BGK collision integral [10] is accompanied by large errors. These features of ion drift play an important role when considering the properties of cryogenic discharges and in a mixture of gases [5, 13-15]. The above numerical data explain the results of the analysis of ion-atom collisions [16] and significantly supplement the reference data [1-4].

\section{References}

1. E. W. McDaniel, E. A. Mason. The Mobility and Diffusion of Ions in Gases // Wiley. - New York. - P. 1973.
2. H.W. Ellis, R.Y. Pai, E.W. McDaniel, E.A. Mason, L.A.Viehland // Atomic Data and Nuclear Data Tables. - 1976. - Vol. 17. - No 3. - P. 177. 
3. E.W. McDaniel, L.A.Viehland. The transport of slow ions in gases: Experiment, theory, and applications // Physics Reports. - 1984. - Vol. 110. No. 5-6. - P. 333.

4. E.A. Mason, L.A.Viehland // Atomic Data and Nuclear Data Tables. - 1995. - Vol. 60. - No. 1. - P. 37.

5. S.A. Maiorov. Ion drift in a gas in an external electric field // Plasma Phys. Rep. - 2009. - Vol. 35. - P. 802.

6. S. A. Maiorov, V. N. Tsytovich, B. Lebedev. On the accuracy of the BGK model for ion drift in own gas // Phys. Inst. - 2012. - Vol. 39. - P. 72.

7. R.I. Golyatina, S.A. Maiorov. Approximation of the characteristics of ion drift in parent gas // Plasma Physics Reports. - 2017. - Vol. 43. - No. 1. - P. 75.

8. B.M. Smirnov. // Physics of Ionized Gases. Wiley-VCH. - 2001

9. G.A. Bird. // Molecular gas dynamics. - Oxford University Press. -1976.

10. D. Else, R. Kompaneets, S. V. Vladimirov. // Phys. Plasmas. - 2009. - Vol. 16. - P. 062106.

11. M. Lampe, T. B. Röcker, G. Joyce, S. K. Zhdanov, A. V. Ivlev, G. E. Morfill. Ion distrbution function in a plasma with uniform electric field // Phys. Plasmas. 2012. - Vol. 19. - P. 113703.

12. L. A. Sena. Inelastic Collisions of Light Atoms in the Adiabatic Approximation // JETP. - 1946. - Vol. 16. - P. 734;

13. S.A. Maiorov, T.S. Ramazanov, K.N. Dzhumagulova, A.N. Jumabekov, A.N.Dosbolaev. Investigation of plasma-dust structures in He-Ar gas mixture // Phys. Plasm. - 2008. - Vol. 15. - P. 093701.

14. S. N. Antipov, E. I. Asinovskii, A. V. Kirillin, S. A. Maiorov, V. V. Markovets, O. F. Petrov, V. E. Fortov. Charge and Structures of Dust Particles in a Gas Discharge at Cryogenic Temperatures // JETP. - 2008. Vol. 106. - P. 830.

15. S. N. Antipov, M. M. Vasil'ev, S. A. Maiorov. O. F. Petrov, V. E. Fortov. Dusty plasma structures in He-Kr DC glow discharge //JETP. - 2011. - Vol. 112. P. 482.

16. D. Piscitelli, A.V. Phelps, J. Urquijo, E. Basurto, L.C. Pitchford. Ion mobilities in $\mathrm{Xe} / \mathrm{Ne}$ and other rare-gas mixtures // Phys. Rev. E. - 2003. - Vol. 68. - P. 046408. 